Proc. IEEE VTC 2004 Fall

\title{
Layout Design for Multiple Collocated Wireless Mesh Networks
}

\author{
Pai-Hsiang Hsiao and H. T. Kung \\ Division of Engineering and Applied Sciences \\ Harvard University \\ Cambridge, MA 02138 \\ \{shawn, htk\}@eecs.harvard.edu
}

\begin{abstract}
We consider a fundamental problem in wireless mesh networking that concerns the layout of access points (APs) of multiple simultaneously operable wireless mesh networks collocated in the same region. For given directional antennas, we are interested in dense and uniform layouts that will maximize the number of these wireless mesh networks without introducing radio interference, while providing convenient access to any of them from any location. We describe an optimal layout method, called the "diagonal placement scheme", for wireless meshes of squares under a simplifying interference model. Then we show upper and lower bounds for the performance of any optimal layout without the simplifying assumption. Our solutions have applications in a number of areas, including QoS routing, secure routing, fault-tolerance, and load balancing.
\end{abstract}

\section{INTRODUCTION}

Wireless mesh networking [1] based on $802.11 \mathrm{a} / \mathrm{b} / \mathrm{g}$, 802.16a or other technologies represents an emerging area of interest in wireless infrastructures. For example, in January 2004, IEEE approved formation of a mesh study group for 802.11 protocols, and in June 2004, the 802.11s task group was formed. The 802.16a standard includes support for wireless mesh architecture. While these wireless mesh networks hold great promise in providing relatively inexpensive and rapidly deployable network infrastructure, they pose new challenges in areas such as QoS routing, secure routing, fault-tolerance, and load balancing.

Use of multiple simultaneously operable networks collocated in the same region is a well-known technique in addressing these challenges. For example, in supporting QoS, we can direct all high-priority real-time traffic to a specific wireless network dedicated to such traffic. Similarly, in providing secure access to an external gateway or application server, one of these wireless networks with strong encryption can serve as a dedicated access network. These multiple networks also represent a powerful networking infrastructure for supporting fault-tolerance and load balancing.

When deploying these wireless networks in the same physical area, radio interference is the major concern. To avoid interference, we could partition the available radio channel into sub-channels and limit each network to a designated sub-channel. In this paper, we consider an alternative method where multiple networks can share the same channel simultaneously by using directional antennas (traditional, steerable or smart antenna systems). To support simultaneous operations of these wireless networks, their access points (APs) will need to be carefully laid out in order to avoid interference. For a given transmit beam pattern of the directional antenna, we define the wireless mesh layout problem to be the problem of packing as many simultaneously operable wireless mesh networks as possible in the same physical region, while providing convenient access to any of these wireless mesh networks from any location.

We make several simplifying assumptions in this paper. We assume that wireless mesh networks are identical 2dimensional $W \times H$ meshes of squares, with the edges of each square being of unit length. For short, we will call these wireless mesh networks meshes. Associated with each square of a mesh, there are four APs one on each of its corners. We assume that all APs produce the same transmit beam pattern. To avoid interference, unintended receivers cannot lie in the area of a transmit beam pattern. We consider two beam patterns: simplified rectangular and ideally sectorized, as illustrated in Fig. 1. The length of the beam sector is $1+\epsilon$ where $\epsilon$ is a small positive number. The gain inside the beam pattern is constant and is negligible both outside the pattern and on the boundary.

The rest of the paper is organized as follows. In Section II, we show an optimal layout method, called the diagonal placement scheme, assuming the simplified rectangular beam pattern. In Section III, we use the results from Section II

(a)

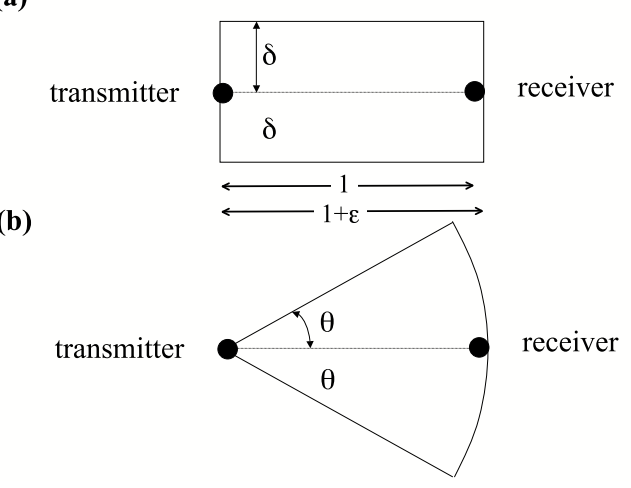

Fig. 1. Two transmit beam patterns: (a) simplified rectangular beam pattern, and (b) ideally sectorized beam pattern 
to derive upper and lower bounds for the wireless mesh layout problem assuming the ideally sectorized beam pattern, and in addition, we formulate a mathematical optimization problem for the layout problem. In Section IV we describe an application of our layout solution in load balancing routing for wireless access networks. We conclude in Section V.

\section{Optimal Layout fOR Simplified Rectangular BEAM PATTERNS}

In this section, we describe an optimal layout method, called the diagonal placement scheme (DPS), for the wireless mesh layout problem assuming the simplified rectangular beam pattern.

The simplified rectangular beam pattern as depicted in Fig. 1(a) is a rectangular area of width $2 \delta$ and height $1+\epsilon$, centered along the line connecting the transmitter and the receiver. Being a coarse approximation of beam patterns in the real world, the simplified rectangular beam pattern greatly simplifies the characterization of the interference area and, as a result, allows a relatively simple proof on the optimality of DPS.

Fig. 2 shows an exemplary layout of four $4 \times 4$ meshes, each using 25 APs. We use different shapes to denote APs belonging to different meshes, with squares, circles, diamonds, and triangles representing APs of the 0-th, first, second, and third network, respectively. The figure also depicts the bounding area, which is defined to be the minimal size rectangle that encloses all the APs. Note that a wireless terminal at any location in this environment has convenient access to all the four meshes, in the sense that the terminal has local access to their APs.

As mentioned in Section I, the goal of the layout design is to pack as many simultaneously operable networks as possible while providing convenient access from any location. We say a layout is a feasible layout if it produces no interference, i.e., every transmit beam pattern covers exactly two APs, the transmitter and the intended receiver. Furthermore, we say a layout is an optimal layout if it is a feasible layout and it packs the most number of networks with the same bounding area.

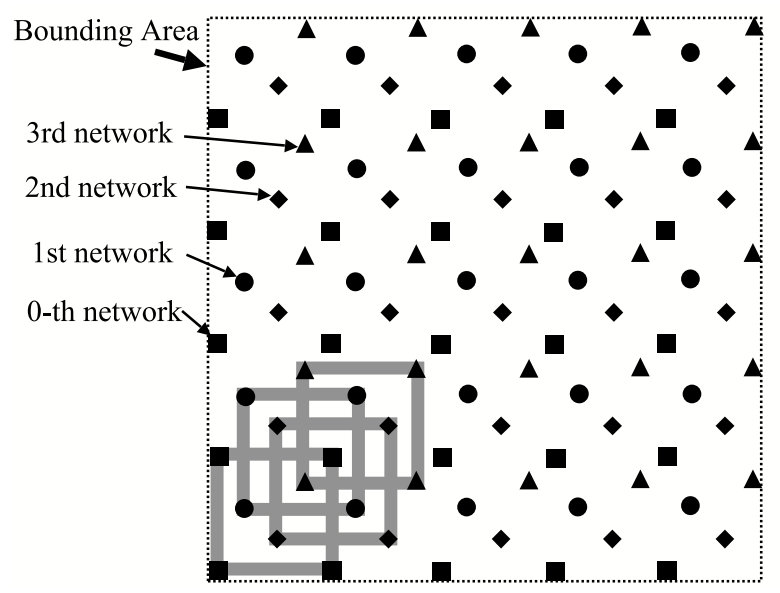

Fig. 2. An exemplary layout for four $4 \times 4$ meshes
Because these networks are identical meshes of squares, we need only inspect a fraction of their APs to ensure that it is a feasible layout. In particular, for each mesh we focus on the four APs of its bottom-leftmost square as shown in Fig. 2. We note that for any optimal layout, the bottom-leftmost squares of any two meshes must intersect, for otherwise we could reduce the bounding area by shifting one mesh to intersect with the other without introducing interference. Thus, without loss of generality, we represent a layout design by the displacement of each of the meshes relative to the bottom-leftmost mesh (e.g., 0 -th network shown in Fig. 2). More precisely, we represent a layout design for $N$ meshes by $\left(X_{i}, Y_{i}\right)$, where $0 \leq i<N$, $X_{0}=Y_{0}=0$ and $0 \leq X_{i}, Y_{i}<1$.

\section{A. Diagonal Placement Scheme}

Assume that $\delta<\frac{1}{N}$ where $\delta$ is the beam width as depicted in Fig. 1(a). We consider the layout design for $N$ meshes where, for $1 \leq i<N$, the $i$-th mesh has a displacement of $(i \times \delta, i \times \delta)$. Since the displacements are all on a diagonal, we call this layout the diagonal placement scheme (DPS) for $N$ meshes. It is clear that DPS is a feasible layout. In subsection II-C below, we will show that DPS is an optimal layout. For an example of the DPS layout, see the layout of Fig. 3 at the end of the third round.

Given a beam width of $\delta$, DPS can pack at most $\left\lfloor\frac{1}{\delta}\right\rfloor$ meshes. On other hand, in order to pack $N$ meshes, $\delta$ cannot be greater than $\frac{1}{N}$.

\section{B. The Diagonalization Process}

We describe a process, called diagonalization, which can transform any feasible layout into the DPS layout. This process consists of $N$ rounds, with $N$ being the number of meshes. For each round, a base mesh is chosen before the round starts and is retired from the process after the round ends. We choose the base mesh to be the mesh that has the smallest $X$ and $Y$ displacements among all the remaining meshes. We will show that there is always a base mesh at the beginning of each round. As shown in Fig. 3, a round consists of three steps: tighten, swap and release. The first step, tighten, shifts all remaining meshes, excluding the base mesh, simultaneously towards left and bottom as much as possible without introducing interference. More precisely, we first reduce all $X$ displacements of the remaining meshes, excluding the base mesh, by the same amount as much as possible without introducing interference. We then reduce the $Y$ displacements without introducing interference. Since the tighten step does not introduce interference, the layout remains to be feasible after the step.

After the tighten step, among the remaining meshes, excluding the base mesh, we find the meshes with the smallest $X$ and $Y$ displacements. If there is a single mesh that has both the smallest $X$ and $Y$ displacements, then we enter the release step; otherwise, we perform the swap step. Suppose that the displacements of the two meshes found are $\left(X_{p}, Y_{p}\right)$ and $\left(X_{q}, Y_{q}\right)$, where $X_{p}<X_{q}$ and $X_{p}>Y_{q}$. In this case, the swap step moves one of the two meshes to $\left(X_{p}, Y_{q}\right)$ and the 


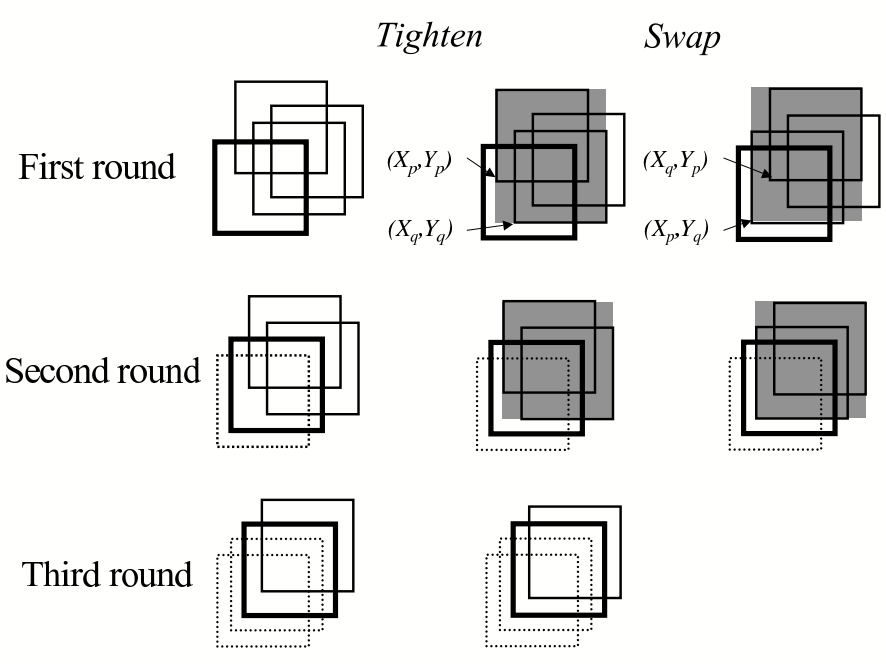

Fig. 3. Diagonalization process on a layout of four meshes. For each round the current base mesh is drawn in bold lines, and the retired base meshes are drawn in dashed lines.

other to $\left(X_{q}, Y_{p}\right)$. Now the mesh that relocates to $\left(X_{p}, Y_{q}\right)$ has both the smallest $X$ and $Y$ displacements, and we can continue to the release step. We note that the swap step does not introduce interference, so the layout remains to be feasible after the step.

Finally, in the release step, the base mesh is retired, and among the remaining meshes, the one with the smallest $X$ and $Y$ displacement becomes the next base mesh. Future rounds will not consider the retired meshes. Fig. 3 illustrates the diagonalization process on a layout of four meshes.

Lemma 1: The diagonalization process transforms any given feasible layout into the DPS layout without increasing the bounding area.

Proof: In each round, after the tighten step, the smallest possible $X$ and $Y$ displacements of the remaining meshes are both $\delta$ relative to the base mesh. The swap step ensures that there will be a mesh with a $(\delta, \delta)$ displacement from the current base mesh after the current round, and it becomes the next base mesh. Thus, the displacement of the next base mesh is always $(\delta, \delta)$ from the current base mesh. As a result, the displacement of $j$-th round's base mesh is $((j-1) \times \delta,(j-$ $1) \times \delta)$. This means that the diagonalization process transforms the given layout into the DPS layout.

To show that the diagonalization process does not increase the bounding area, we note first that both tighten and release steps do not increase the bounding area by the definitions of their operations. We note next that the swap step does not change the bounding area. Before the swap step, the displacements of the two meshes with the smallest $X$ and $Y$ displacements are $\left(X_{p}, Y_{p}\right)$ and $\left(X_{q}, Y_{q}\right)$ with $X_{p}<X_{q}$ and $Y_{p}>Y_{q}$. Suppose that the meshes are $W \times H$ meshes of squares. Then the upper and right boundaries of the bounding rectangle for these two meshes have their $Y$ and $X$ coordinates equal $Y_{p}+H-1$ and $X_{q}+W-1$, respectively. After the swap step, the displacements of the two meshes are $\left(X_{p}, Y_{q}\right)$ and $\left(X_{q}, Y_{p}\right)$. The upper and right boundaries of the bounding (a)

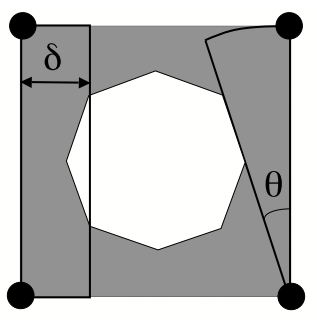

(b)

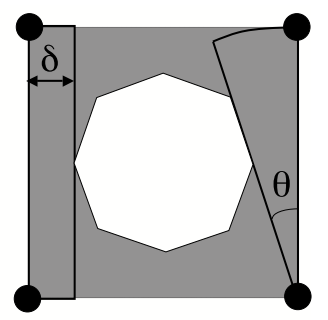

Fig. 4. Representing the ideally sectorized beam pattern using the simplified rectangular beam pattern. The shaded areas are the union of eight transmit beam patterns among the four APs, assuming the ideally sectorized beam pattern. The two representations by the simplified rectangular beam pattern: (a) the worst-case representation, and (b) the best-case representation

rectangle for the two meshes with the new displacements still have their $Y$ and $X$ coordinates equal to $Y_{p}+H-1$ and $X_{q}+W-1$, respectively.

\section{Optimality of the Diagonal Placement Scheme}

Theorem 1: The DPS layout is an optimal layout under the simplified rectangular beam pattern assumption.

Proof: Given any optimal layout, by Lemma 1, the diagonalization process can transform the layout into the DPS layout without increasing the bounding area. Thus, the DPS must be an optimal layout.

\section{LAyOut PRoblem FOR IDEALly SECTORIZED BEAM PATTERNS}

In this section, we address the layout problem for the ideally sectorized beam pattern (Fig. 1(b)). Frequently used in the literature to model directional antennas [2]-[4], this beam pattern is generally considered a better approximation to real-world beam patterns than the simplified rectangular beam pattern.

We will first derive lower and upper bounds for the performance of any optimal layout by applying the results of Section II. We then formulate the layout problem as a mathematical optimization problem and use numerical optimization tools, such as MATLAB, to compute numerical solutions.

Fig. 4 depicts two interference-area representations for the ideally sectorized beam pattern, based on the simplified rectangular beam pattern. The shaded area is the union of eight ideally sectorized beam patterns among the four APs. Fig. 4(a) shows the worst-case representation. It uses the narrowest simplified rectangular beam patterns of which the union can cover the entire shaded area. Because all feasible layouts using this representation are also feasible layouts using the ideally sectorized beam pattern, this representation allows us to establish a lower bound for the performance of the ideally sectorized beam pattern. Fig. 4(b) shows the best-case representation. It uses the widest simplified rectangular beam patterns of which the union does not exceed the shaded area. Because all feasible layouts using the ideally sectorized beam pattern are also feasible layouts for this representation, we can use this representation to establish an upper bound for the performance of the ideally sectorized beam pattern. 


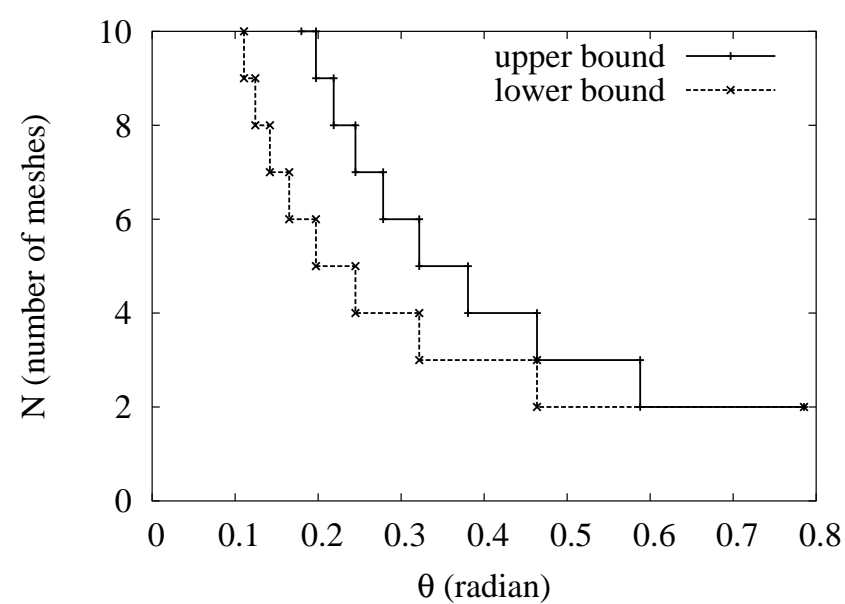

Fig. 5. Upper and lower bounds on the number of meshes that can be packed as a function of beam angle $\theta$ for the ideally sectorized beam pattern

Given a beam angle $\theta$ (see Fig. 1(b)), the beam widths $\delta$ (Fig. 1(a)) of the worst-case and best-case representation are $\frac{\tan \theta}{1+\tan \theta}$ and $\frac{\tan \theta}{2}$, respectively. Based on the $\delta$ values, we can compute lower and upper bounds for the bounding area size for the ideally sectorized beam pattern using the results of the previous section.

In addition, we can derive a lower bound of $\arctan \left(\frac{1}{N-1}\right)$ and an upper bound of $\arctan \left(\frac{2}{N}\right)$ on the maximum beam angle $\theta$ that allows a feasible layout of $N$ meshes, respectively. With these results, we can bound the number of meshes that can be packed given a beam angle $\theta$, as shown in Fig. 5 . Note that the gap between the upper and lower bounds on the number of allowed meshes decreases as the beam angle $\theta$ increases.

Having established the upper and lower bounds, we now turn our attention to the formulation of the layout problem as a mathematical optimization problem. To model the interference constraints imposed by the transmit beam, we define an interference distance function $i d f\left(\left(x_{1}, y_{1}\right),\left(x_{2}, y_{2}\right),\left(x_{3}, y_{3}\right)\right)$, where $\left(x_{i}, y_{i}\right)$ are locations. The function returns a value less than $\theta$ if location $\left(x_{3}, y_{3}\right)$ lies in the transmit beam from $\left(x_{1}, y_{1}\right)$ to $\left(x_{2}, y_{2}\right)$; otherwise, it returns a value that is greater than or equal to $\theta$. More specifically, let $U=\left(x_{2}-x_{1}, y_{2}-y_{1}\right)$ and $V=\left(x_{3}-x_{1}, y_{3}-y_{1}\right)$. That is, $U$ is the vector from $\left(x_{1}, y_{1}\right)$ to $\left(x_{2}, y_{2}\right)$ and $V$ is the vector from $\left(x_{1}, y_{1}\right)$ to $\left(x_{3}, y_{3}\right)$. The function returns $\arccos \left(\frac{U \cdot V}{\|U\|\|V\|}\right)$, the angle between the two vectors, if $\|V\| \leq 1$, and it returns $2 \pi \times\|V\|$ if $\|V\|>1$. ( $U \cdot V$ denotes the inner product of $U$ and $V$, and $\|V\|$ the norm of $V$ )

We can formulate the layout problem as a non-linear optimization problem as follows:

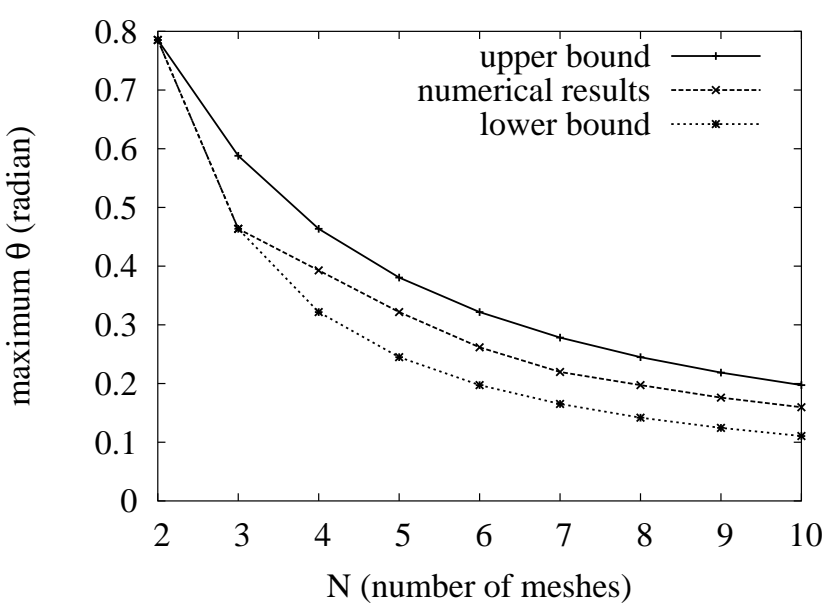

Fig. 6. Lower bound, upper bound, and best-effort numerical result for the maximum angle as a function of $\mathrm{N}$, for the ideally sectorized beam pattern

$$
\begin{aligned}
& \operatorname{minimize}\left(W+\max X_{i}\right) \times\left(H+\max Y_{i}\right) \\
& \text { subject to } \\
& -i d f\left(\left(X_{j}, Y_{j}\right),\left(X_{j}+1, Y_{j}\right),\left(X_{k}, Y_{k}\right)\right)+\theta \leq 0 \\
& -i d f\left(\left(X_{j}+1, Y_{j}\right),\left(X_{j}, Y_{j}\right),\left(X_{k}, Y_{k}\right)\right)+\theta \leq 0 \\
& -i d f\left(\left(X_{j}, Y_{j}\right),\left(X_{j}, Y_{j}+1\right),\left(X_{k}, Y_{k}\right)\right)+\theta \leq 0 \\
& -i d f\left(\left(X_{j}, Y_{j}+1\right),\left(X_{j}, Y_{j}\right),\left(X_{k}, Y_{k}\right)\right)+\theta \leq 0 \\
& -i d f\left(\left(X_{j}+1, Y_{j}\right),\left(X_{j}+1, Y_{j}+1\right),\left(X_{k}, Y_{k}\right)\right)+\theta \leq 0 \\
& -i d f\left(\left(X_{j}+1, Y_{j}+1\right),\left(X_{j}+1, Y_{j}\right),\left(X_{k}, Y_{k}\right)\right)+\theta \leq 0 \\
& -i d f\left(\left(X_{j}, Y_{j}+1\right),\left(X_{j}+1, Y_{j}+1\right),\left(X_{k}, Y_{k}\right)\right)+\theta \leq 0 \\
& -i d f\left(\left(X_{j}+1, Y_{j}+1\right),\left(X_{j}, Y_{j}+1\right),\left(X_{k}, Y_{k}\right)\right)+\theta \leq 0 \\
& 0 \leq X_{i}, Y_{i}<1
\end{aligned}
$$

where $\theta$ is the beam angle and $0 \leq i<N, 0 \leq j, k<N$, $j \neq k$.

The optimization problem minimizes the size of the bounding area, subject to the constraints of interference. The interference constraints are expressed as eight inequalities for each pair of distinct meshes. A pair of meshes, say $J$ and $K$, are represented by $\left(X_{j}, Y_{j}\right)$ and $\left(X_{k}, Y_{k}\right)$. Each of the eight inequalities corresponds to one of the eight beams on mesh $J$, and represents constrains where $K$ can be placed. Thus, there are a total of $8 \times N \times(N-1)$ such constraints.

This formulation allows a numerical method to compute locally optimal solutions only, which are not necessarily globally optimal. The computed results will depend on the initial values of $\left(X_{i}, Y_{i}\right)$.. In our computation, we use multiple initial values for $\left(X_{i}, Y_{i}\right)$, and use the lower bound and upper bound derived from the simplified rectangular beam pattern to help evaluate the quality of the computed numerical solutions.

By making $\theta$ a variable and changing the objective function to maximize $\theta$ instead of minimizing bounding area size, we can obtain numerical solutions to the maximum beam angle for packing $N$ meshes. Fig. 6 plots the lower and upper bounds on the maximum $\theta$, as well as best-effort numerical results, for the modified optimization problem, as a function of $N$. For each $N$, we compute numerical solutions for the problem 


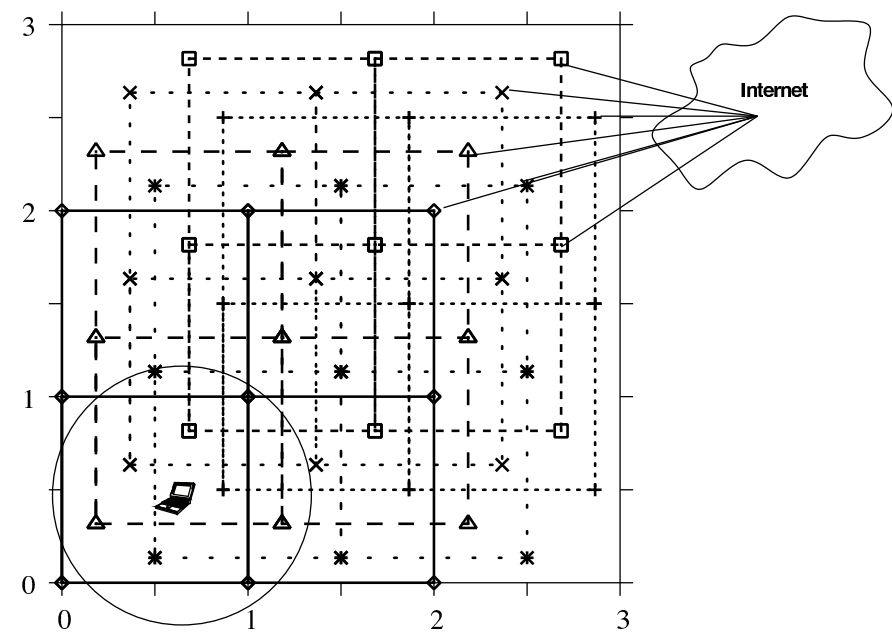

Fig. 7. An exemplary layout of six $2 \times 2$ meshes. The six APs in the upper rightmost corners are connected to the Internet via an egress node. The wireless terminal, denoted by a laptop, at the lower-left corner can access the APs of all six meshes within its radio range

10,000 times with each time starting with a random initial value for $\left(X_{i}, Y_{i}\right)$, and the best solution is reported as the numerical result.

Fig. 7 shows the layout with the largest maximum beam angle we obtained using numerical methods for six $2 \times 2$ meshes. The maximum beam angle computed is 0.2618 (radian), while the lower and upper bounds derived using result of Section II are 0.1974 and 0.3218 , respectively.

In this section, we described a general approach for solving the wireless mesh layout problem. When dealing with complicated beam patterns, it could be difficult to solve the problem analytically. We show how to formulate the layout problem as a mathematical optimization problem and obtain solutions better than the lower bound by using a numerical optimization solver.

\section{LOAD-BALANCING ROUTING FOR WIRELESS ACCESS NETWORKS: AN APPLICATION}

We describe an application of the result of this paper for a special class of wireless networks, termed wireless access networks. Via such a network, an end node, such as a wireless terminal, can send and receive packets from an egress node connected to the external networking infrastructure.

Suppose that the egress node has wired connections to several APs, and that the bandwidth of the external connection of the egress node exceeds the total bandwidth of these APs. As pointed out in [5], a challenge in this case is to load balance the use of these APs to maximize the bandwidth use of the external connection. Suppose that there are six APs that have wired high-speed connections to the egress node. Then we can stipulate that each of these APs is connected to a separate mesh as shown in Fig. 7. Whenever an end node, e.g., the laptop shown in Fig. 7, needs to send data to the external world, it will randomly select one of the several meshes to use. In this way, load balancing is automatically achieved.

\section{CONCLUSION}

In this paper, we motivate and introduce a layout problem for wireless mesh networks with directional antennas. To the best of our knowledge, this is the first piece of work that addresses the layout problem of multiple wireless networks using directional antennas. This layout problem is important both because wireless mesh networks represent an emerging type of network infrastructure, and because the use of multiple collocated and simultaneously operable meshes can potentially address several of challenges in wireless mesh infrastructure such as QoS, security and load-balancing routing.

An optimal layout for wireless meshes of squares is described under the assumption of the simplified rectangular beam patterns. More specifically, the diagonal placement scheme (DPS) generates an optimal layout. This layout scheme is optimal in the sense that it packs the most number of meshes for a given bounding area. We then use this result to establish lower and upper bounds for the solution to the same problem under the assumption of ideally sectorized beam patterns. A similar approach can be adapted to analyzing the layout problem with other complicated beam patterns. We formulate a non-linear optimization problem to obtain numerical solutions to the layout problem with ideally sectorized beam patterns. While the numerical method does not guarantee that it will find a globally optimal solution for this mathematical formulation, the formulation is general and can be extended to other beam patterns.

\section{ACKNOWLEDGMENT}

This research was supported in part by NSF grant \#ACI0330244, and in parts by research grants from Microsoft Research and Sun Microsystems.

\section{REFERENCES}

[1] D. Beyer, "Wireless mesh networks for residential broadband," in $\mathrm{Na}$ tional Wireless Engineering Conference, San Diego, 2002.

[2] Y.-B. Ko, V. Shankarkumar, and V. N. H., "Medium access control protocols using directional antennas in ad hoc networks," in IEEE INFOCOM, 2000.

[3] M. Takai, J. Martin, A. Ren, and R. Bagrodia, "Directional virtual carrier sensing for directional antennas in mobile ad hoc networks," in ACM International Symposium on Mobile Ad Hoc Networking and Computing (MOBIHOC), 2002.

[4] J. E. Wieselthier, G. D. Nguyen, and A. Ephremides, "Engery-limited wireless networking with directional antennas: The case of session-based multicasting," in IEEE INFOCOM, 2002.

[5] P.-H. Hsiao, A. Hwang, H. T. Kung, and D. Vlah, "Load-balancing routing for wireless access networks," in IEEE INFOCOM, 2001. 